\title{
Variations de la production et de la composition du lait au pâturage en fonction des conditions climatiques
}

\author{
P D'Hour, JB Coulon \\ INRA, laboratoire adaptation des herbivores aux milieux, \\ Theix, 63122 Saint-Genès-Champanelle, France
}

(Reçu le 19 avril 1993; accepté le 7 juin 1993)

\begin{abstract}
Résumé - Vingt-deux vaches laitières de type Pie noire ou de race Montbéliarde ont été conduites ensemble en pâturage continu pendant $76 \mathrm{j}$, à $1100 \mathrm{~m}$ d'altitude. La production et la composition chimique du lait ont été mesurées tous les jours, de même que les caractéristiques climatiques. Des températures moyennes comprises entre 15 et $20^{\circ} \mathrm{C}$ ont été associées à des productions de lait supérieures à $0,5 \mathrm{~kg} / \mathrm{j}(P<0,01)$ à celles observées lorsque les températures étaient comprises entre 4 et $10^{\circ} \mathrm{C}$, mais à des taux butyreux et protéique inférieurs d'environ $1 \mathrm{~g} / \mathrm{kg}(P<0,05)$. Ces effets disparaissent presque complètement lorsque la pluviométrie est importante (supérieure à $5 \mathrm{~mm} / \mathrm{j}$ ). Ces réponses sont identiques chez les vaches Pies noires et Montbéliardes.
\end{abstract}

vache laitière / climat / composition du lait

Summary - Effect of climate on milk production and composition. Twenty-two Holstein or Montbéliarde dairy cows were pastured on a continuous grazing system for $76 d$ in the Auvergne. Climatic conditions, milk production and fat and protein contents were measured every day. Milk yield was higher $(+0.5 \mathrm{~kg} / \mathrm{d}, \mathrm{P}<0.01)$ when the mean temperature ranged between 15 and $20^{\circ} \mathrm{C}$ than when it ranged between 4 and $10^{\circ} \mathrm{C}$. This difference disappeared when the rainfall was higher than $5 \mathrm{~mm} / \mathrm{d}$. In contrast, the fat and protein contents were lower (about $-1 \mathrm{~g} / \mathrm{kg}, \mathrm{P}<0.05$ ) when the mean temperature was between 15 and $20^{\circ} \mathrm{C}$. There was no difference in these responses of $\mathrm{Hol}-$ stein and Montbéliarde cows.

dairy cow / climate / milk composition

\section{INTRODUCTION}

Les variations d'un jour à l'autre de la production et de la composition du lait des vaches laitières au pâturage peuvent être importantes, mais leur analyse est rendue délicate en raison des nombreux facteurs qui peuvent intervenir et de l'importance et de la durée de ces variations. Ainsi, si l'on connait assez bien les effets de la 
conduite du pâturage sur les variations de la production laitière (Hoden et al, 1987), les liaisons entre ces variations et les conditions climatiques en milieu tempéré sont encore à définir. Le climat peut en effet intervenir directement (limitation de la capacité d'ingestion, stress thermique des animaux) ou indirectement et à moyen terme (baisse de la quantité d'herbe disponible et de sa qualité) sur la production et la composition du lait. Si ces effets ont fait l'objet de nombreux travaux en milieu tropical (Hancock, 1954 ; Thatcher, 1974 ; Rodriguez et al, 1985 ; Berbigier, 1988 ; Sharma et al, 1988), ce n'est pas le cas en milieu tempéré (cf revue de Rémond et Vermorel, 1982).

L'objectif de cette étude a été, dans des conditions d'altitude où les variations climatiques d'un jour à l'autre peuvent être importantes, de décrire précisément les variations de la production et de la composition du lait de vaches laitières conduites sur une même parcelle durant toute la durée de l'essai, et d'analyser ces variations en fonction des variations climatiques.

\section{MATÉRIEL ET MÉTHODES}

\section{Animaux et alimentations}

Vingt-deux vaches multipares du domaine de Marcenat (Cantal, $1100 \mathrm{~m}$ d'altitude) pour moitié de type Holstein et pour l'autre moitié de race Montbéliarde, ont été utilisées pendant $76 j$ de pâturage (16 mai - 30 juillet). Leur production laitière à la mise à l'herbe (11 mai) était comprise entre 18 et $37 \mathrm{~kg} / \mathrm{j}(25,5 \mathrm{~kg} / \mathrm{j}$ en moyenne) et leur stade de lactation entre 30 et $190 \mathrm{j}$ (128 $\mathrm{j}$ en moyenne). Pendant les $10 \mathrm{j}$ qui ont suivi la mise à l'herbe, elles ont reçu $4 \mathrm{~kg} / \mathrm{j}$ de foin, offert durant les traites, réalisées à l'étable, en place, le matin entre $6 \mathrm{~h}$ et $8 \mathrm{~h}$, et le soir entre $15 \mathrm{~h} 15$ et $17 \mathrm{~h} 15$.

Elles ont été conduites sur une seule parcelle de prairie naturelle, située à $1500 \mathrm{~m}$ de l'étable, à un chargement de 3,1 vaches/ha. On a considéré que le pâturage couvrait les besoins d'entretien, plus ceux nécessaires à la production de $20 \mathrm{~kg}$ de lait, et on a complémenté les animaux à raison de $t \mathrm{~kg}$ de concentré par tranche de $2,5 \mathrm{~kg}$ de lait au-dessus de cette production. Les quantités de concentré distribuées ont été prédéterminées en prenant pour référence la production des semaines -1 et -2 avant la mise à l'herbe et en admettant une décroissance de la production laitière de $2,5 \%$ par semaine. La réduction des apports de concentrés à la mise à l'herbe a été réalisée à raison de $3 \mathrm{~kg}$ par semaine et a été terminée en $2 \mathrm{sem}$ au plus.

\section{Mesures}

Les quantités de lait produites ont été pesées tous les jours. Le taux butyreux et le taux protéique ont été mesurés tous les jours, à chaque traite. Les quantités ingérées d'aliment concentré ont été mesurées tous les jours.

Chaque semaine, un prélèvement d'herbe a été réalisé (en 5 endroits différents, chacun d'une surface de $0,8 \mathrm{~m}^{2}$ ) en début d'après-midi et analysé. La quantité d'herbe offerte est passée de 1,3 t MS/ha à la fin du mois de mai à 0,8 tha à la fin du mois de juillet. Parallèlement, les teneurs en matières azotées totales et en cellulose brute sont passées de 17,4 et $20,3 \%$ MS à 15,1 et $25,7 \%$ MS. Ces mesures ont permis de confirmer que ni la quantité d'herbe offerte ni sa qualité n'ont été un facteur limitant de la production laitière.

Dans une station météorologique standard située à proximité de l'étable, la température sous abri, la pluviométrie, la durée d'ensoleillement et la vitesse moyenne du vent ont été relevées quotidiennement.

\section{Analyse des données}

Pour mettre en évidence l'effet propre du climat sur les performances des animaux, on a calculé, pour chaque jour, l'écart entre la valeur observée moyenne par race (pour la production laitière, le taux butyreux et le taux protéique, et les quantités de matières corespondantes) et celle obtenue par régression sur les 76 j de pâturage. 
La moyenne de ces écarts sur les jours $j$ et $j+1$ (qui a permis d'obtenir les meilleurs résultats comparativement à la valeur des écarts des jours j ou j+1) a ensuite été étudiée par analyse de variance en introduisant dans le modèle la race (Pie noire ou Montbéliarde) et les caractéristiques climatiques du jour j regroupées en 3 classes : pluviométrie (nulle, comprise entre 0 et $5 \mathrm{~mm}$, supérieure à $5 \mathrm{~mm}$ ) et moyenne des températures maximale et minimale (inférieure à $10^{\circ} \mathrm{C}$, comprise entre 10 et $15^{\circ} \mathrm{C}$, et supérieure à $15^{\circ} \mathrm{C}$ ). La durée d'ensoleillement, initialement introduite dans le modèle, n'ayant jamais présenté d'effet significatif, n'a pas été retenue dans les analyses définitives.

\section{RÉSULTATS}

\section{Climat}

Au cours de l'essai, la pluviométrie a été importante $(272 \mathrm{~mm}$ au total) et relativement fréquente $(55 \%$ de jours avec pluie, bien répartis sur la période) (fig 1). Les températures moyennes sous abri ont été comprises entre 4 et $22^{\circ} \mathrm{C}\left(11,9^{\circ} \mathrm{C}\right.$ en moyenne). La température minimale n'est descendue au-dessous de $0^{\circ} \mathrm{C}$ que $2 \mathrm{j}$, au cours de la première semaine de l'essai. La température maximale n'a jamais dépassé $27^{\circ} \mathrm{C}$. II n'y a pas eu de liaison significative entre la pluviométrie et la température moyenne. Au cours de l'essai, la vitesse moyenne du vent est restée relativement faible (comprise entre 1 et $3 \mathrm{~m} / \mathrm{s}$ en moyenne sur $24 \mathrm{~h}$ ). La durée d'ensoleillement a varié de 0 à $15 \mathrm{~h} / \mathrm{j}(6,7 \mathrm{~h} / \mathrm{j}$ en moyenne).

\section{Évolution de la production et de la composition du lait}

La production laitière s'est accrue avec la température $(+0,5 \mathrm{~kg} / \mathrm{j}$ entre les 2 classes extrêmes ; $P<0,01$ ), et dans une moindre

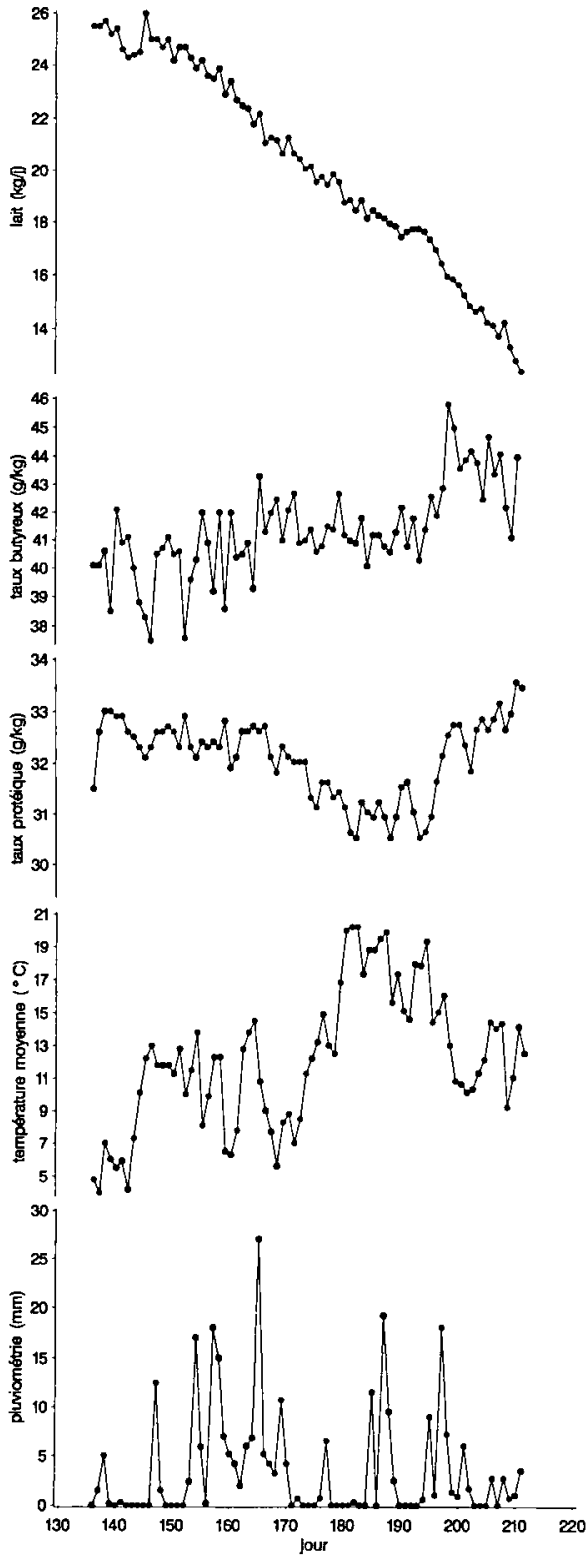

Fig 1. Évolution de la production et de la composition du lait (moyenne des 22 vaches) et des conditions climatiques au cours de la période expérimentale. 
Tableau I. Effet de la température moyenne et de la pluviométrie sur les performances des vaches laitières (écarts à la régression sur les $76 \mathrm{j}$ de l'essai).

\begin{tabular}{|c|c|c|c|c|c|c|c|c|c|}
\hline & \multicolumn{4}{|c|}{ Température $\left({ }^{\circ} \mathrm{C}\right)$} & \multicolumn{4}{|c|}{ Pluviométrie $(\mathrm{mm})$} & \multirow[t]{2}{*}{ ETR a } \\
\hline & $<10$ & $10-15$ & $>15$ & & 0 & $0-5$ & $>5$ & & \\
\hline Nombre de jours & 22 & 37 & 17 & & 34 & 21 & 21 & & \\
\hline Température moyenne $\left({ }^{\circ} \mathrm{C}\right)$ & 7,2 & 12,4 & 18,0 & & 12,7 & 11,3 & 12,2 & & \\
\hline Pluviométrie (mm) & 3 & 4 & 4 & & 0 & 2 & 11 & & \\
\hline Lait $(\mathrm{kg} / \mathrm{j})$ & $-0,2$ & 0,0 & 0,3 & $* \star$ & $-0,1$ & 0,1 & 0,2 & * & 0,5 \\
\hline Taux butyreux $(\mathrm{g} / \mathrm{kg})$ & 0,3 & 0,2 & $-0,6$ & * & $-0,3$ & 0,1 & 0,1 & NS & 1,2 \\
\hline Taux protéique $(\mathrm{g} / \mathrm{kg})$ & 0,2 & 0,3 & $-0,9$ & $*$ & $-0,2$ & 0,0 & $-0,1$ & NS & 0,5 \\
\hline Matières grasses $(\mathrm{g} / \mathrm{j})$ & 1 & -2 & 4 & NS & -2 & 1 & 4 & NS & 22 \\
\hline Matières protéiques $(\mathrm{g} / \mathrm{j})$ & -4 & 7 & -1 & $* *$ & -6 & 5 & 4 & $* *$ & 12 \\
\hline
\end{tabular}

a Écart type résiduel. "* $P<0,01 ;$ * $P<0,05$.

mesure, avec la pluviométrie $(+0,3 \mathrm{~kg} / \mathrm{j}$ lorsque la pluviométrie était supérieure à $5 \mathrm{~mm}$ comparativement aux jours sans pluie ; $P<0,05$ ) (tableau I). L'effet des températures élevées n'a été important que lorsqu'elles n'étaient pas associées à de la pluie $(+0,7 \mathrm{~kg} / \mathrm{j}$ en l'absence de pluie contre $+0,1 \mathrm{~kg} / \mathrm{j}$ lorsque la pluviométrie était supérieure à $5 \mathrm{~mm}$, interaction significative ; $P<0,01$ ).

La composition chimique du lait n'a été affectée que par la température, et de manière inverse de la production laitière: les températures élevées ont eu un effet défavorable sur les taux butyreux $(-0,9 \mathrm{~g} / \mathrm{kg}$ entre les températures les plus élevées et les plus faibles, $P<0,05$ ) et protéique $(-1,1 \mathrm{~g} / \mathrm{kg}, P<0,01)$. En définitive, la production de matières utiles a été très peu affectée par les conditions climatiques (tableau I) ; la production de matières protéiques semble cependant un peu supérieure lorsque la température est comprise entre 10 et $15^{\circ} \mathrm{C}(+10 \mathrm{~g} / \mathrm{j}$ environ comparativement aux températures inférieures à $10^{\circ} \mathrm{C}$ ou supérieures à $15^{\circ} \mathrm{C}$ ).
Les variations de la production et de la composition du lait sous l'effet des facteurs climatiques ont été identiques chez les animaux de type Pie noire et chez ceux de race Montbéliarde.

\section{CONCLUSION}

Cette étude montre que, même dans des conditions tempérées, les performances des vaches laitières peuvent être affectées, de manière limitée mais significative, par les conditions climatiques, et en particulier la température. Dans les conditions de cet essai, où les vaches sont pratiquement toujours restées dans leur zone de confort thermique $\left(-5\right.$ à $21^{\circ} \mathrm{C}$ de température moyenne; Johnson, 1987b), la production de lait semble favorisée lorsque la température se situe entre 15 et $20^{\circ} \mathrm{C}$, contrairement aux taux de matières utiles. Au-delà de $25^{\circ} \mathrm{C}$, on sait que la température a un effet défavorable sur la production (Thatcher, 1974; Johnson, 1987b). Ces observations vont dans le même sens 
que celles faites par Huth et Schutzbar (1980) dans des conditions voisines. Cet effet des conditions climatiques n'est vraisemblablement pas un effet indirect, par le biais notamment de la qualité et de la disponibilité de l'herbe, qui sont restées élevées durant l'essai, et qui auraient dû, en modifiant les apports nutritifs, affecter la production de matières utiles. II s'agit donc plutôt d'un effet physiologique direct et à très court terme (24 h), modifiant, comme c'est le cas pour la photopériode (Tucker, 1985), la dilution des matières sécrétées. Les causes de cet effet restent à préciser (Johnson, 1987a et b), de même que l'effet légèrement favorable de la pluviométrie sur la production laitière que nous avons observé. D'autre part, au pâturage, d'autres facteurs climatiques peuvent intervenir en addition et/ou en interaction avec la température, comme cela a été mis en évidence ici avec la pluviométrie, et par d'autres auteurs pour le rayonnement solaire, la vitesse de l'air et l'hygrométrie (Johnson, 1987b; Huth et Schutzbar, 1980). Ces travaux doivent être complétés par l'étude des variations individuelles de réaction des animaux aux modifications des conditions climatiques.

\section{RÉFÉRENCES}

Berbigier P (1988) Bioclimatologie des ruminants domestiques en zone tropicale. INRA, Paris, $237 p$
Hancock J (1954) The direct influence of climate on milk production. Dairy Sci Abstr 16, 90-102

Hoden A, Fiorelli JL, Huguet L, Muller A, Weiss $P$, Jeannin B (1987) Le pâturage simplifié pour les vaches laitières. Bull Tech CRZV Theix INRA 69, 55-65

Huth FW, Schutzbar WV (1980) Der Einfluss des Wetters auf die Milchleistung der Kühe während des Weideganges. Tierzuchter 32 , 16-23

Johnson HD (1987a) Bioclimate and livestock. In: Bioclimatology and the adaptation of livestock (HD Johnson, ed) Elsevier, Amsterdam, 3-16

Johnson HD (1987b) Bioclimate effects on growth, reproduction and milk production. In: Bioclimatology and the adaptation of livestock (HD Johnson, ed) Elsevier, Amsterdam, 35-57

Rémond B, Vermorel M (1982) Influence du climat et de la saison sur la production laitière au pâturage. In : Actions du climat sur l'animal au pâturage. INRA, Paris, 115-129

Rodriguez LA, Mekonnen G, Wilcox CJ, Martin FG, Krienke WA (1985) Effects of relative humidity, maximum and minimum temperature, pregnancy, and stage of lactation on milk production and yield. $J$ Dairy Sci 68, 973-978

Sharma AK, Rodriguez LA, Wilcox CJ, Collier RJ, Bachman KC, Martin FG (1988) Interactions of climatic factors affecting milk yield and composition. J Dairy Sci 71, 819-825

Thatcher WW (1974) Effects of season, climate, and temperature on reproduction and lactation. J Dairy Sci 57, 360-368

Tucker HA (1985) Photoperiodic influences on milk production in dairy cows. In: Recent advances in animal nutrition (WE Haresign, DJA Cole, eds) Butterworths, 211-221 